\title{
MECHANICAL LOADING AND HOW IT AFFECTS BONE CELLS: THE ROLE OF THE OSTEOCYTE CYTOSKELETON IN MAINTAINING OUR SKELETON
}

\author{
J. Klein-Nulend ${ }^{1, *}$, R.G. Bacabac ${ }^{2}$ and A.D. Bakker ${ }^{1}$ \\ ${ }^{1}$ Department of Oral Cell Biology, ACTA-University of Amsterdam and VU University Amsterdam, Research \\ Institute MOVE, Amsterdam, The Netherlands \\ ${ }^{2}$ Department of Physics, Medical Biophysics Group, University of San Carlos, Cebu City, Philippines
}

\begin{abstract}
Lack of physical activity causes bone loss and fractures not only in elderly people, but also in bedridden patients or otherwise inactive youth. This is fast becoming one of the most serious healthcare problems in the world. Osteocytes, cells buried within our bones, stimulate bone formation in the presence of mechanical stimuli, as well as bone degradation in the absence of such stimuli. As yet, we do not fully comprehend how osteocytes sense mechanical stimuli, and only know a fraction of the whole range of molecules that osteocytes subsequently produce to regulate bone formation and degradation in response to mechanical stimuli. This dramatically hampers the design of bone loss prevention strategies. In this review we will focus on the first step in the cascade of events leading to adaptation of bone mass to mechanical loading, i.e., on how osteocytes are able to perceive mechanical stimuli placed on whole bones. We will place particular emphasis on the role of the osteocyte cytoskeleton in mechanosensing. Given the crucial importance of osteocytes in maintaining a proper resistance against bone fracture, greater knowledge of the molecular mechanisms that govern the adaptive response of osteocytes to mechanical stimuli may lead to the development of new strategies towards fracture prevention and enhanced bone healing.
\end{abstract}

Keywords: Mechanical loading; mechanotransduction; fluid shear stress; osteocyte; bone cell; cytoskeleton; inflammatory cytokines; cell mechanics; cell shape; cell stiffness.

*Address for correspondence:

J. Klein-Nulend

Department of Oral Cell Biology

Research Institute MOVE,

ACTA-VU University Amsterdam, Gustav Mahlerlaan 3004 ,

1081 LA Amsterdam, The Netherlands

Telephone Number: +31205980881

FAX Number +31205980333

E-mail j.kleinnulend@acta.nl
Bones adapt to mechanical loading

Throughout life bone is constantly remodelled by the coordinated action of bone-resorbing osteoclasts and bone-forming osteoblasts in basic multicellular units. This continuous remodelling likely serves to prevent and remove fatigue-related micro-damage and allows adaptation of the bone mass and structure. The balance between the amount of bone resorption and bone formation determines whether the process of bone remodelling leads to a net gain or loss of bone mass. The number and activity of osteoclasts and osteoblasts are determined by a multitude of factors, such as hormones and cytokines, as well as by locally produced signalling molecules under the influence of mechanical stimuli (Vezerides et al., 2006; You et al., 2008; Onal et $a l ., 2012)$. The osteocyte is a source of soluble factors not only to target cells on the bone surface but also to target distant organs, such as muscle, kidney, and other tissues (Bonewald, 2011).

During physical activity, mechanical forces are exerted on the bones through ground reaction forces and by the contractile activity of muscles (Lanyon et al., 1975; Usui et al., 2003). These physical forces result in a maintenance or gain of bone mass, but also drive adaptation of bone structure. The adaptation of trabecular bone architecture according to the demands of mechanical usage is evident in the vertebrae, where the trabeculae are predominantly oriented in the longitudinal direction, providing the best possible resistance to compression fracture of the vertebrae with a minimal use of material. A classic example of the stimulating effect of mechanical stimuli on bone mass is provided by the bones in the forearm of tennis players. The ulna and radius in the arm that holds the racket are exposed to high impact forces, leading to tiny deformations in the stiff bone matrix and an increase in bone mass of 5 to $10 \%$ compared to the ulna in the contra-lateral arm (Ducher et al., 2004). The deformations that occur in bones as a result of physical forces are expressed as strain, where 1,000 microstrain equals a $0.01 \%$ change in length of the bone compared to its original length. Vigorous exercise induces bone strains up to 1,000 microstrain in humans (Lanyon et al., 1975). By comparison, controlled bouts of whole bone loading resulting in 1,000 to 3,000 microstrain are anabolic in experimental animal models of bone-loading, demonstrating the potential for appropriate physical exercise routines as a means to enhance bone mass (Rubin and Lanyon, 1987; Turner et al., 1994; Reijnders et al., 2007). 
In contrast with the increase in bone mass with vigorous physical exercise, we lose bone mass in the absence of mechanical stimuli. Bone mass is rapidly lost under unloading conditions, e.g., during bed rest, hind-limb unloading in mice, or local denervation of muscles (Globus et al., 1986; Vandamme et al., 2012). Interestingly, strains resulting from habitual activity suffice to prevent bone loss compared to complete unloading, even though these habitual strains hardly ever exceed 400 microstrain. Thus, either mechanical loads that lead to tiny strains within the bone matrix are somehow sensed by the cells within bone, which then act to preserve bone mass, or sporadic mechanical stimuli that exceed 2,000 microstrain still occur often enough to prevent bone loss. We have shown that cultured bone cells release nitric oxide (NO) in response to mechanical stimulation in the form of a fluid shear stress, and that the amount of NO released linearly correlates to the rate of the applied fluid shear stress (Bacabac et al., 2004). Since the rate at which a mechanical stimulus is applied is the product of the magnitude (amplitude) and the frequency of the stimulus, this supports the notion that low-magnitude, high-frequency mechanical stimuli are as potent in evoking a response in bone cells as high-amplitude, low-frequency stimuli (Ozcivici et al., 2010). In other words, very small mechanical stimuli may elicit a cellular response only if applied fast enough. The rate dependent-response to stress provides a possible explanation why adaptive bone formation in vivo may occur despite the sporadic occurrence of high-amplitude strains in daily life. The question how daily mechanical loads preserve bone mass is clinically highly relevant, as with our ageing population more and more people suffer from fragile bones.

\section{Osteocytes sense mechanical stimuli and direct mechanical adaptation of bone}

The cells likely responsible for sensing the physical stimuli derived from mechanical forces exerted on bones are the osteocytes, which comprise over $90 \%$ of the bone cells. Osteocytes are stellate cells that are embedded within the calcified bone matrix. They form a large number of cellcell contacts through their long slender cell processes, forming a syncytium capable of rapid transduction of signals (Fig. 1). Osteocytes are highly mechanosensitive, likely more so than periosteal fibroblasts or osteoblasts, and alter the production of a multitude of signalling molecules when triggered by a mechanical stimulus. Mechanically activated osteocytes produce signalling molecules like bone morphogenetic proteins (BMPs), Wnts, prostaglandin $\mathrm{E}_{2}\left(\mathrm{PGE}_{2}\right)$, and $\mathrm{NO}$, which can modulate the recruitment, differentiation, and activity of osteoblasts and osteoclasts (Robling et al., 2006; Tan et al., 2007; You et al., 2008; Santos et al., 2009 ). Thus, osteocytes are theoretically capable of orchestrating bone adaptation in response to mechanical stimuli. That osteocytes are indeed essential mediators of osteoclastic bone resorption was confirmed in an elegant experiment by Tatsumi et al. (2007). They showed that the loss of bone mass following hind limb unloading of mice was prevented when $\sim 80 \%$ of the osteocytes were ablated. Osteocytes thus seem to stimulate osteoclast activity in the absence of daily mechanical loads, a capability that has been confirmed in in vitro studies (You et al., 2008; Kulkarni et al., 2010). Indeed it has been shown recently by two independent groups that RANKL production by osteocytes determines bone mass in adult mice, demonstrating the importance of osteocytes in the regulation of bone mass (Nakashima et al., 2011; Xiong et al., 2011). Interestingly, the same study demonstrating the requirement of osteocytes for mediating unloadinginduced bone loss also showed that the anabolic response of bone to (re)loading does not require the presence of living osteocytes (Tatsumi et al., 2007). However, this does not eliminate the role of osteocytes in mediating the anabolic response of bone to loading under normal conditions.

\section{Fluid flow, strain, and hydrostatic pressure as stimuli for osteocytes}

If osteocytes are the professional mechanosensing cells of bone, then how do these cells sense whole bone loads? One popular theory entails that matrix strains surrounding the osteocyte cell processes drive a thin layer of extracellular fluid surrounding the osteocyte cell processes to flow across a pressure gradient. This flow of fluid "amplifies" local strains, and is thereby the mechanical signal that is ultimately sensed by the osteocytes. There is ample experimental evidence to support the idea that deformations of the bone matrix drive an interstitial fluid flow. Knothe-Tate et al. have shown experimentally a flow of extra-cellular fluid around the osteocytes as a result of bone tissue strains, by loading of sheep tibiae and following the distribution of tracers through the lacuno-canalicular network (Knothe-Tate et al., 1998; Knothe-Tate et al., 2000). More recently, Price et al. (2011) used fluorescence recovery after photobleaching for imaging fluid displacement synchronised with mechanical loading, to show that the mechanical loading of mouse tibia enhanced fluid transport through the lacuno-canalicular system, demonstrating the correlation of canalicular fluid flow with mechanical load. In addition, several investigators reported that it is not the amount of strain applied to a whole bone that influences bone formation, but the rate at which the strain is applied (Price et al., 2011). Dynamic bone loading, which enhances fluid flow, has also been demonstrated to induce an osteogenic response (Lanyon and Rubin, 1984; Luo et al., 1995; Mosley and Lanyon, 1998). Static loading on the other hand has little effect on lacuno-canalicular fluid flow, and has only a minor effect on bone formation (Lanyon and Rubin, 1984). It has been extensively demonstrated that osteocytes in vitro are sensitive to a flow of fluid when seeded as a monolayer on flat, 2-dimensional (2D) substrates (Klein-Nulend et al., 1995a; Klein-Nulend et al., 1995b; Ajubi et al., 1996; Bakker et al., 2001; Bacabac et al., 2004; Bakker et al., 2009; Litzenberger et al., 2010; Juffer et al., 2012; Kulkarni et al., 2012). One could argue that interstitial fluid is driven to flow within the canaliculi over only osteocyte processes in vivo while a laminar fluid flow over cells seeded on a flat substrate will deform the cell body as well as the cell 

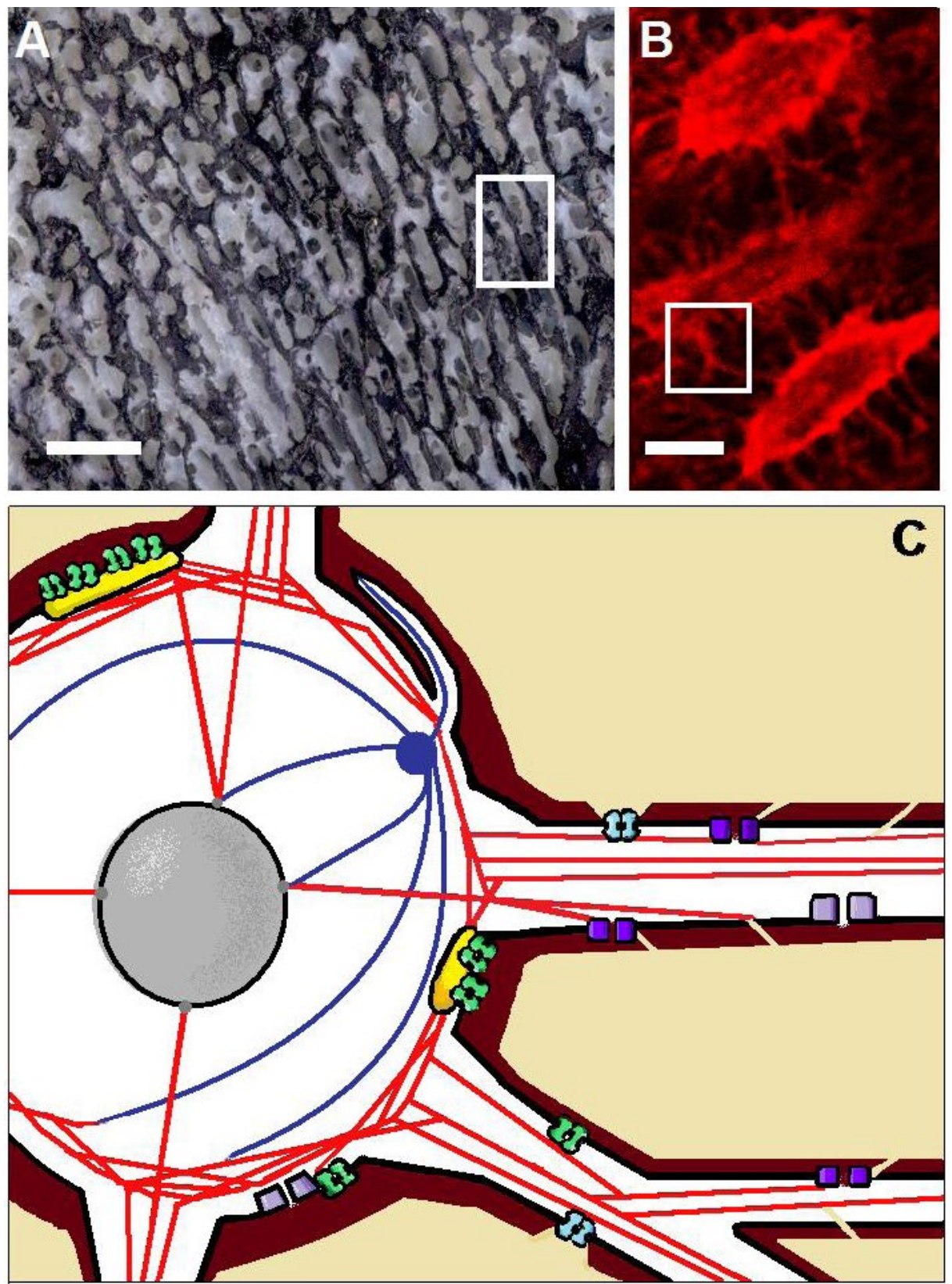

Fig. 1. In osteocytes, all is connected. (A) Macroscopic photograph of a transversely sectioned human femoral head, dipped in black ink to highlight the orientation of the bone trabeculae. Scale bar indicates $5 \mathrm{~mm}$. (B) High magnification osteocytes embedded within their matrix. The osteocytes with their many cell processes are visible (red). Scale bar indicates $5 \mu \mathrm{m}$. (C) Schematic drawing of part of an osteocyte cell body with processes (white) residing within the calcified matrix (ECM; beige). The osteocyte cell membrane is surrounded by interstitial fluid and proteoglycans (ECM; brown). Microtubules (blue lines) originate from the centrosome and form a scaffold along which numerous molecules shuttle. Microtubules also form the core of the primary cilium. Osteocyte cell processes contain mostly actin (red lines), cross-linked by fimbrin (not shown) at places where processes bifurcate. The processes contain $\alpha v \beta 3$ integrins (blue), possibly located on top of collagen "hillocks", and protein tethers. These tethers may deform as a result of interstitial fluid flow and subsequently "tug" at the actin cytoskeleton, or pull open stretch-activated ion channels (purple), allowing calcium and other ions to enter the cell and activate a multitude of chemical signalling cascades. The fluid flow may also tug directly at integrins, such as $\alpha 5 \beta 1$, present along the cell processes and body and associated with hemi-channels. Activated hemi-channels release signalling molecules such as ATP, triggering a signalling cascade. The mechanical signal may be transduced from sites where integrins are triggered, via the actin cytoskeleton, to distant sites, including the nucleus, which is connected to actin via LINC complexes. Forces applied to actin fibres can likely also open stretch-activated ion channels. Integrins are often clustered together at focal adhesions (yellow), which in osteocytes are predominantly located at places where the cell processes intersect with the cell body. Focal adhesions contain tyrosine kinases, such as focal adhesion kinase, which make focal adhesions a prime location for transducing mechanical signals into a chemical response. Considering that the cytoskeleton interconnects virtually every part of the mechanosensing machinery, one can easily imagine that changes in cytoskeletal properties affect mechanosensing. 
processes, thereby eliciting a response that otherwise would not be provoked (McGarry et al., 2005a; Fritton and Weinbaum, 2009). However, it has been shown recently using a Stokesian fluid stimulus probe that electrical signalling is provoked at much lower forces when applied to a cell process of osteocytes compared to the cell body, thus suggesting that in vitro fluid flow experiments provide a valid confirmation of the sensitivity of osteocytes to fluid flow (Wu et al., 2011).

In addition to responding to strain-driven fluid flow, it is also possible that osteocytes respond to matrix strains directly. MC3T3-E1 osteoblasts increase their production of signalling molecules in response to substrate deformations as low as 3,400 microstrain (Robinson et al., 2006), and osteocytes are more mechanosensitive than osteoblasts (Klein-Nulend et al., 1995a). The relative flat and spread shape of isolated bone cells in 2D culture may greatly hamper their sensitivity to a mechanical stimulus (Bacabac et al., 2008), and strains that are not able to elicit a response in bone cells adhered to a flat and stiff surface may be perfectly able to elicit a response in cells in their natural 3-dimensional (3D) conformation. Thus, direct sensation of bone strains may already suffice to activate mechanosensitive bone cells in vivo. It has also been proposed that matrix strains can be locally amplified up to 3-fold by the inhomogeneities in the matrix that are formed by the osteocyte lacunae (Bonivtch et al., 2007). Matrix strains around the osteocyte cell bodies may thus exceed whole bone strains, especially at points where cell processes intersect with the cell body, and could be sufficient to directly activate the osteocytes (Bonivtch et al., 2007). In this regard the observation by Vatsa et al. (2008a), that molecules involved in cellular mechanotransduction such as F-actin and paxillin are concentrated at these intersections, is highly interesting (Fig. 1).

Bones can be considered as a material containing large (vasculature) and small (canaliculi) interconnected fluidfilled pores. The permeability of the lacuno-canalicular porosity is several orders of magnitude lower than that of the vascular porosity (Gardinier et al., 2010). Since the lacuno-canalicular porosity has a low permeability, rapidly placed load on bone causes strains that first pressurise the interstitial fluid around the osteocytes, and then drive fluid flow causing dissipation of the build-up of hydraulic pressure (Wang et al., 1999). The magnitude of pressure experienced by osteocytes in vivo may reach up to $5 \mathrm{MPa}$ according to recent calculations (Gardinier et al., 2010). Cyclic hydraulic pressures of $68 \mathrm{kPa}$ can modulate signalling molecule production in cells of the mouse MLO-Y4 osteocyte cell line, and a pressure of $13 \mathrm{kPa}$ was sufficient to stimulate prostaglandin production by primary osteocytes isolated from chicken calvariae (Klein-Nulend et al., 1995a; Liu et al., 2010). This suggests that, besides substrate strain and fluid shear stress, the loading-induced hydraulic pressure could potentially serve as a mechanical stimulus for osteocytes. Whatever the mechanical load-derived stimulus is that activates the osteocytes, the question remains which osteocyte feature enables the perception of the physical stimulus and subsequent transduction into a chemical signal. This question is not necessarily restricted to osteocytes, as eukaryotic cells in general are sensitive to mechanical stress.

\section{Cellular features enabling mechanotransduction in osteocytes}

A multitude of sensory elements exist that allow cells to detect mechanical stimuli. Mechanosensing is enabled by force-induced conformational changes in cellular structures, such as stretch-activated ion channels, integrin complexes, and cell-cell adhesions. The conformational changes enable the influx and efflux of ions or the activation of signalling cascades, resulting in altered cell shape and altered activity and production of proteins (Hoffman et al., 2011). The cytoskeleton, which can be considered a composite gel-like material (of actin, microtubules, intermediate filaments and their crosslinkers) is the scaffold determining cellular shape and stiffness (Sugawara et al., 2008). Molecules like integrins anchor to the extracellular matrix (ECM) and mechanically link the exterior of the cell to the cytoskeleton, forming trans-membrane complex structures. These complexes, often clustered in so-called focal adhesions, are therefore prime suspects as mechanotransducers.

With respect to osteocyte mechanosensing, the focal adhesion kinase inhibitor-14 has been shown to abolish fluid flow-induced stabilisation of $\beta$-catenin and consequent activation of the $\mathrm{Wnt} / \beta$-catenin pathway in osteocytes, suggesting that focal adhesions and integrins play an important role in osteocyte mechanosensing (Santos et al., $2010)$. Indeed, $\beta 1$-integrins on osteocytes in vivo have been shown to mediate specific aspects of mechanotransduction, confirming the importance of integrins for mediating mechanical stimuli in osteocytes (Litzenberger et al., 2010). Interfering with $\beta 1$-integrin signalling in vitro reduced the upregulation of cyclooxygenase- 2 normally observed after mechanical stimulation of osteocytes, but did not affect mechanically induced intracellular calcium mobilisation (Litzenberger et al., 2010). In addition, $\alpha 5 \beta 1$ integrins interact directly with connexin 43 (Cx43), and this interaction is required for mechanical stimulationinduced opening of Cx43-containing hemi-channels (Batra et al., 2012). Cx43-containing hemi-channels are readily expressed on osteocytes and affect the response of osteocytes to mechanical loading in vivo (Zhang et al., 2011). Direct mechanical perturbation of $\alpha 5 \beta 1$ integrins leads to the opening of the $\mathrm{Cx} 43$-containing hemi-channels (Batra et al., 2012). Integrin attachments thus likely serve as the mechanotransducing units that potentiate the opening of hemichannels (Burra et al., 2010). Other integrins that may mediate osteocyte mechanotransduction are $\alpha v \beta 3$ integrins. Although $\alpha v \beta 3$ integrins are not essential for the attachment of osteocytes to ECM in vitro (Aarden et al., 1996), they may play a role in osteocyte physiology, since it has been shown recently that $\alpha v \beta 3$ integrins mediate signalling via DMP-1, a molecule that is almost exclusively produced by osteocytes (Wu et al., 2011). Interestingly, in vivo $\alpha v \beta 3$ integrins are present along the 
osteocyte processes which are thought to be the main sites of mechanotransduction in osteocytes (McNamara et al., 2009). The glycocalyx of the osteocyte dendritic process is required for the formation of strong integrin attachments (Burra et al., 2010; Burra et al., 2011), demonstrating that the role of integrins should be considered in the context of the complex structures in which they reside before we can fully comprehend how mechanical stimuli are transduced to osteocytes. It should also be noted that as a consequence of osteocytes expressing their own specific set of integrins and the selectivity of integrins for substrates, the nature of the ECM likely affects the mechanoresponse of osteocytes.

Although stretch-activated ion channels have long been suspected to play a role in osteocyte mechanotransduction, it is currently unclear which molecules act as stretchactivated ion channels in osteocytes. The osteocyte response to mechanical loading can clearly be inhibited by gadolinium chloride, which is a non-specific blocker of TRP channels (Ajubi et al., 1999; Bakker et al., 2009). However, which member of the extensive family of TRP channels is responsible for transducing mechanical signals in the osteocyte has not been elucidated. It is unlikely that TRPV6 is a candidate as it is only present at low levels in murine osteoblasts and osteocytes and plays a minor functional role in calcium uptake by osteoblasts (Little et al., 2011).

To understand how the cellular features mentioned above act as a mechanotransduction complex, one needs to regard the cellular context of these features. It would be difficult, if not impossible, for forces acting on a cell to induce conformational changes in cellular molecules that are freely floating in a semi-liquid cell membrane. However, structures that are anchored to neighbouring cells, the ECM or the glycocalyx, as well as to the cytoskeleton, form a direct mechanical link between the extracellular environment and the intracellular compartment (Fig. 1). Such structures are in an excellent position to sense mechanical forces. Integrins are coupled to the cytoskeleton via molecules such as vinculin, talin, and $\alpha$-actinin. Though non-trivial, one may imagine three non-linear springs in a series representing the mechanical link between the ECM, the transmembrane proteins (including the focal adhesions), and the cytoskeleton (Fig. 2), to conceptualise the transfer of forces between these protein structure clusters (Fig. 3). Note, however, that each protein network is expected to become stiffer in response to applied forces (Storm et al., 2005), which could support an amplified force transfer. Hence, the importance of anchoring mechanotransduction complexes that connect the ECM to the cytoskeleton predicts that the osteocyte cytoskeleton plays a key role in osteocyte mechanotransduction.

\section{Structure of the osteocyte cytoskeleton}

The cytoskeleton is a scaffold made out of protein components that provide mechanical structure to cells. The viscoelastic properties of the cytoskeletal structure provide cells with resistance to shear or compression, enable cell

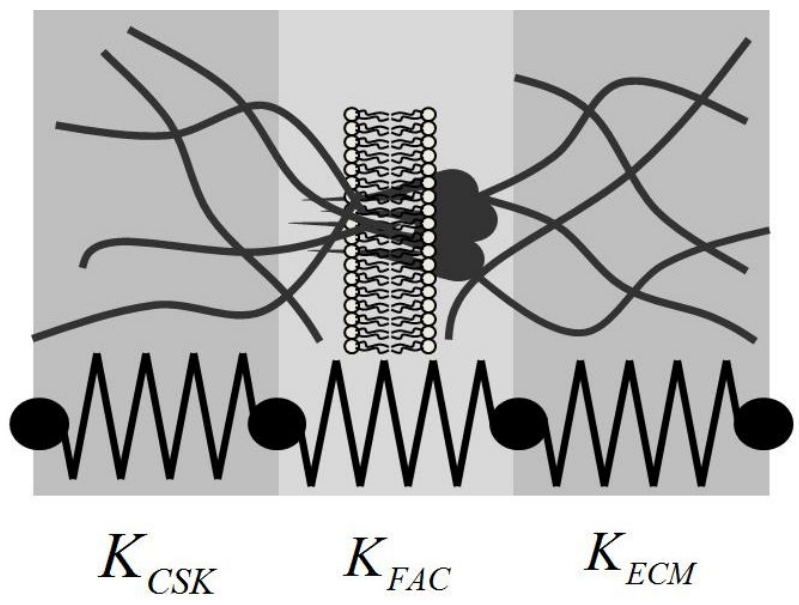

Fig. 2. Spring-series conceptualisation of mechanical linkage between the cytoskeleton (CSK), focal adhesion complexes (FAC), and the extracellular matrix (ECM), with respective non-linear spring constants,

$K_{C S K}, K_{F A C}, K_{E C M}$.

migration, enable transport of intracellular molecules, determine the mechanical properties of the cells, and allow for mechanosensing. Eukaryotic cells contain three main kinds of cytoskeletal structures, each built up out of their own proteins: actin, intermediate filaments, and microtubules. The distribution of each of these structures seems to change when osteoblasts differentiate into osteocytes. In parallel, the stiffness of osteoblasts decreases during their differentiation towards osteocytes (Sugawara et al., 2008). Microtubules are limited in distribution to the proximal region of osteocyte processes but extend the entire length of cell processes of MC3T3-E1 osteoblasts grown in 3D in collagen gels (Murshid et al., 2007). Microtubules are essential for the integrity and formation of osteoblast cell processes grown in $3 \mathrm{D}$, but processes of primary osteocytes in 3D are dependent on actin (Murshid et al., 2007). Actin filaments are also crucial for maintaining the shape of primary chicken osteocytes when grown on flat substrates (Tanaka-Kamioka et al., 1998). Not surprisingly, osteocytes also contain a set of actin-bundling proteins distinctive from that in osteoblasts. Fimbrin and $\alpha$-actinin are predominantly present in the processes of osteocytes, with especially strong signals of fimbrin at the sites of bifurcation of the processes (Kamioka et al., 2004). Compared to osteoblasts, osteocytes also contain high amounts of villin, which is present within the osteocyte cytoplasm but not within the processes. Osteoblasts immunostained with anti-spectrin show punctate signals on their cytoplasmic membranes, whereas spectrin is co-localised with actin from the distal portion of the cytoplasmic processes to the cell centre (Kamioka et al., 2004). The typical morphology of the osteocyte, determined by its cytoskeleton, was originally thought to be imposed on differentiating osteoblasts during their incorporation in the bone matrix. Osteocytes have to remain in contact with other cells and ultimately with the bone surface to ensure access to oxygen and nutrients. Culture 


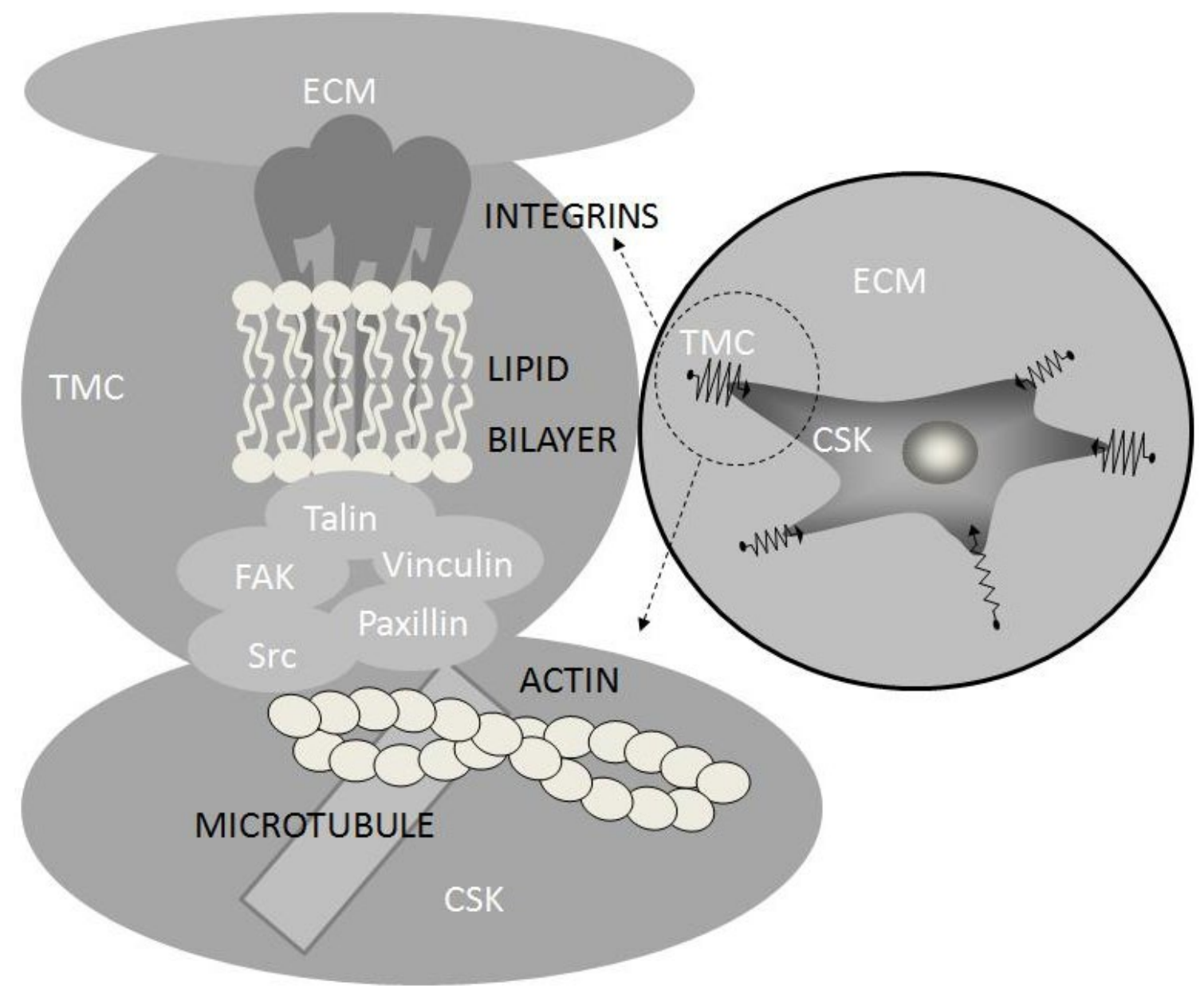

Fig. 3. Protein network clusters across the extracellular matrix, trans-membrane proteins, and the cytoskeleton regions of a spread cell. ECM, extracellular matrix; TMC, transmembrane complex; FAK, focal adhesion kinase.

experiments with isolated chicken osteocytes have shown, however, that although the cells lose their stellate shape in suspension, they re-express this morphology as soon as they settle on a support (Van der Plas and Nijweide, 1992). Apparently, the typical stellate morphology and the need to establish a cellular network are intrinsic characteristics of terminal osteocyte differentiation.

Although the stellate shape of osteocytes seems innate, by no means does this shape represent a fixed or static feature. Dallas and co-workers (Veno et al., 2006) have shown cell body movement and the extension and retraction of cell processes over time using dynamic imaging of living osteocytes within their lacunae. Calvarial explants from transgenic mice with green fluorescent protein expression targeted to osteocytes revealed that the osteocyte is highly dynamic. Therefore the osteocyte processes, rather than being permanent connections between osteocytes as well as between osteocytes and bone surface cells, may have the capacity to connect, disconnect, and re-connect (Bonewald, 2006; Zang et al., 2006). This phenomenon implies a complex role for intercellular connection in bone, and may indicate an adaptive information transfer facility through the ostecytic syncytium beyond mere force transfer mechanisms. Whereas signalling molecule information is diffusion-limited, intercellular communication provides a more efficient means for information transfer.
Since the osteocyte cell processes are of predominant importance for sensing mechanical stimuli, and the osteocyte processes apparently contain mainly actin, the actin cytoskeleton rather than the microtubule cytoskeleton could be important for osteocyte mechanosensing. However, disruption of both the actin cytoskeleton with cytochalasin $\mathrm{B}$ and disruption of the microtubule cytoskeleton with colchicine inhibits mechanical stimulation-mediated release of $\mathrm{PGE}_{2}$ in the mouse osteocyte cell line MLO-Y4 (McGarry et al., 2005b). Besides the cell processes, osteocytes may possess a single primary cilium that contributes to mechanosensing (Malone et al., 2007). The primary cilium is a solitary organelle that emanates from the cell surface of most mammalian cell types, and is a key coordinator of signalling pathways during development and in tissue homeostasis (Berbari et al., 2009). Primary cilia consist of an axoneme of nine doublet microtubules that extends from a basal body. This could explain why disruption of actin as well as of microtubules may upset the osteocyte response to mechanical stimulation. In the mouse osteoblast cell line MC3T3-E1, which has been shown to depend on microtubules rather than actin for their cytoskeletal integrity, inhibition of actin polymerisation did not inhibit intracellular calcium mobilisation or $\mathrm{PGE}_{2}$ release in response to a mechanical stimulus (McGarry et al., 2005b; Malone et al., 2007). In contrast, in calvarial 
osteoblasts derived from chicken, disruption of the actin cytoskeleton did strongly inhibit mechanical loadingstimulated $\mathrm{PGE}_{2}$ release (Ajubi et al., 1996).

\section{Cytoskeletal mechanics and stress-response signatures}

Fluid flow over dendrites in the lacuno-canalicular porosity has been suggested to induce strains in the actin filament bundles of the cytoskeleton that are more than an order of magnitude larger than tissue level strains (You et al., 2001). Using ultrastructural data for the cell process cytoskeleton and the tethering elements that attach the process to the canalicular wall, a 3D model was created for the osteocyte process. Using this model the deformed shape of the tethering elements and the hoop strain on the central actin bundle as a result of loading-induced fluid flow was predicted. It was found that tissue-level strains of $>1,000$ microstrain at $1 \mathrm{~Hz}$ result in a hoop strain of $>0.5 \%$ (You et $a l ., 2001)$. The tethering elements of the osteocyte process can thus act as a strain-amplifier. Tethering filaments appear to be absent in the pericellular space surrounding the cell body, likely due to the wide pericellular space $(\sim 1 \mu \mathrm{m})$ between the cell membrane and the wall of the lacuna, in contrast to the pericellular space surrounding the cell process ( $\sim 80 \mathrm{~nm}$ ) (You et al., 2004). Potentially CD44 serves as the tethering molecule since it is expressed in osteocyte cell processes and has an attachment site for hyaluronan (Noonan et al., 1996). Alternatively, a protein tether involved in transduction of mechanical stimuli has recently been identified in cutaneous mechanoreceptors. This molecule is a protein filament with a length of $\sim 100 \mathrm{~nm}$ (Hu et al., 2010). It is possible that the osteocyte tether is similar to this protein tether.

More recently, a new theoretical model has been developed that predicts that integrin-based attachment complexes along osteocyte cell processes would dramatically and focally amplify small tissue-level strains (Wang et al., 2007). Using rapid fixation techniques it was observed that osteocyte cell processes seem attached directly at canalicular projections emanating from the canalicular wall, via $\alpha \mathrm{v} \beta 3$ integrins (McNamara et al., 2009). The theoretical model predicts that the tensile forces acting on these integrins are $<15 \mathrm{pN}$ and that axial strains caused by the sliding of actin microfilaments about the fixed integrin attachments are an order of magnitude larger than the radial strains in the earlier proposed strain-amplification theory (Wang et al., 2007). In vitro experiments indicated that a Newtonian flow exerting a force of 1 to $2 \mathrm{pN}$ on osteocyte cell processes is able to activate cell signalling to osteocytes, especially when applied around focal contacts (Wu et al., 2011).

To simulate exercise loading on bone tissue, we stimulated bone cells with noisy fluid shear stress and demonstrated that noise enhances the molecular response (Bacabac et al., 2009). An optimum noise intensity stimulated a maximum NO release, which diminished upon the addition of higher intensities (Bacabac et al., 2009). A noise-amplified response implies a threshold-activated mechanism. Thus, despite the meagre strains experienced at normal daily activities, bouts of noisy strains (during strenuous activities as in sports) might provide enough stimuli for enhanced bone cell activity. We also showed in another study that bone cell responses to vibration stress demonstrated a NO release which positively correlated with the cube of the vibration frequency (Bacabac et al., 2006). The response to vibration stress was fundamentally different from the response to fluid shear stress, which evoked a rate-dependent response (i.e., linear to the frequency) (Bacabac et al., 2004). Considering that the cell nucleus is in bulk denser than the cytoplasm containing the cytoskeleton, the cube of the vibration stress stimulation is theoretically linear to the nuclear displacement by momentum transfer, suggesting that the molecular responses observed were linear to nuclear displacement (Bacabac et al., 2006). In other words, the nucleus seemed to vibrate within the cytoplasm. As indicated earlier the extracellular matrix, transmembrane adhesion molecules, and the cytoskeleton can be considered a continuum. This continuum is mechanically coupled to the nucleus via the LINC (linker of nucleoskeleton and cytoskeleton) complex, and forces exerted on integrins by shear stress could be indirectly transferred all the way to the nucleus (Lombardi and Lammerding, 2011; Kardas et al., 2012). Taken together, vibration directly stimulates the cell nucleus, which is probably not the case for fluid shear stress loading. The nucleus thereby contributes to how signalling molecule dosage is controlled by the type of stress sensed, whereas the cytoskeletal structure mediates force transfer (Bacabac et al., 2006).

Several groups suggest that the deformation of the nucleus can be mediated via shear stresses on the cytoskeleton, which in turn might influence gene regulation (Davies et al., 1995; Ingber, 1997; Janmey, 1997). Recently, it has been shown in endothelial cells that nesprin-1, included in the complex linking of the cytoskeleton to the nucleus, is crucial in cytoskeleton-mediated nuclear deformation (Anno et al., 2012). It is therefore implied that for similar shear deformations in osteocytes, nuclear deformation could directly transfer mechanical load to the nucleus by shear stresses on the cytoskeleton, which may re-direct gene expression.

The previous paragraph nicely illustrates that the osteocyte is not just an on-off system, either activated by a mechanical stimulus or not, but rather that it is able to discern different stimuli applied at different frequencies ( $\mathrm{Li}$ et al., 2012a). The highly dynamic nature of the attachment sites and cytoskeleton, which are continuously undergoing turnover, likely explains how mechanical stimuli of varying magnitude and frequency regulate distinct signalling pathways, as elegantly described in a recent review by Hoffman et al. (2011).

Studies on microtubule self-organisation in vitro under microgravity (simulated by clinostats or by space flight), indicate that polymer network structures are fundamentally different compared to microtubules subjected to gravitation (Papaseit et al., 2000). Although conditions within living cells are totally different, such studies could at least provide generic structural tendencies in the presence or absence of gravitation for a network of biopolymers. To characterise networks of biopolymers, the thermal fluctuation of probe 
particles can be monitored using optical tweezers with sub-nm resolution using backfocal plane interferometry techniques (Gittes and Schmidt, 1998; Addas et al., 2004). The mechanical properties of the network can be described by the probe fluctuations when quantified using the power-law of the spectral signature. Considering that the dynamic signature of the mechanical properties of semi-flexible polymer networks is essentially thermal at high frequencies (i.e., the power law of $\mathrm{G}$ above $1 \mathrm{~Hz}$ ), we expect a non-negligible change in the dynamics only at the low frequency end where the non-thermal (or outof-equilibrium) dynamics reflect structure and reaction rates of the underlying molecular processes. A change in the power-law itself (especially at the low frequency end) would indicate a more fundamental rheological change in cellular mechanics. Thus, an experimental approach correlating changes in the effective stiffness of the cells to that of the ECM under different gravitational states is a promising procedure towards understanding how the ECM and the cytoskeleton mechanically relate to each other. If gravitation, or its loss, provides enough stimulus (purely mechanical or biophysical), a biologically meaningful change in the slope of correlation between the stiffness of the cell and that of the ECM should be measurable. In turn, this implies that mechanosensing is either impaired or enhanced depending on the specific cell function.

Recently, we probed the mechano-activity and mechano-sensitivity of various bone cell types and fibroblasts, using a two-particle in vitro assay for measuring the viscoelasticity of cells, with two-particle micro-rheology (Bacabac et al., 2008). Mechano-activity was characterised by the induction of force traction on attachment sites by cells, and mechano-sensitivity is the ability of cells to sense minute forces. We found that osteocytic cell types induce a relatively higher traction force on attached particles than osteoblastic cells. Fibroblastic CCL-224 cells are even more mechano-active compared to MLO-Y4 cells. The force fluctuations on the attached probes reflect intracellular movement, which might include actin (and microtubule) polymerisation, as well as motor and crosslinker dynamics. Since cell migration involves these dynamic processes, the lower magnitude of force fluctuation might reflect a lower capacity of osteocytes for motility compared to fibroblasts (Bacabac et al., 2008).

In a two-particle in vitro assay, MLO-Y4 cells release NO simultaneous with increasing force application (Bacabac et al., 2008). Furthermore, a typical behaviour observed in response to increasing force application was the predictable occurrence of force traction by cells on the attached beads, which was simultaneous with the specific morphological adaptation from a spherical to a polar shape defining ends at the attachment points. It would seem quite clear that force traction, morphology change, and possibly the release of signalling molecules are quantitatively coupled in response to micro-environmental stress conditions. Osteocytes under round-suspended morphology required lower force stimulation in order to show an NO response, even though they were an order of magnitude more elastic compared to flat-adherent cells (Bacabac et al., 2008). Apparently, elastic osteocytes seem to require less mechanical force in order to respond than stiffer cells. On the other hand, flat adherent MLO-Y4 cells, primary chicken osteocytes, MC3T3-E1 osteoblasts, and primary chicken osteoblasts all showed a similar elastic modulus of less than $1 \mathrm{kPa}$ (Bacabac et al., 2008), even though osteocytes are known to be more responsive to mechanical stress than osteoblasts (Klein-Nulend et al., 1995a). This indicates that differences in mechanosensitivity among cells are partly a property of elasticity, which reflects the underlying cytoskeletal structure that supports cell geometry. However, the observation that minute forces $(\sim 5 \mathrm{pN})$ are enough to stimulate soft round cells in contrast with stiff flat ones that require higher forces, suggests that molecular transducers (e.g. ion channels in the membrane) play a role where efficient force transfer across the cytoskeleton is lost (Bacabac et $a l ., 2008)$. Thus, mechanosensing is not solely a property of how cells globally deform under stress but is a complex property that may incorporate the contribution of suspected molecular sensors. The integrity of the cytoskeleton is therefore a prerequisite to mechanosensing mechanisms where intracellular force transfer is required.

Simultaneous with the increased NO release in response to mechanical stimulation, MLO-Y4 osteocytes show increased force traction on the attached beads (Bacabac et al., 2008). The osteocytes generate a force up to $30 \mathrm{pN}$, which is within the order of magnitude capacity of activating integrins. Whether there is a causal link between loading-induced NO production by the cells and force generation is still under investigation. Attachment to opposing colloidal particles, trapped optically, simulates polarised attachment by cells spreading within a modelled ECM (e.g. fibrin or collagen gels), where the trap stiffness corresponds to ECM stiffness (Mizuno et al., 2009). The observation that force fluctuation increased at higher trap stiffness (while cell stiffness remain constant), suggests a mechanism where the cell adapts itself to the stiffness of the surroundings. This adaptation is a direct way by which an osteocyte could "feel" an applied mechanical load. "Feeling" and adapting to the mechanical properties is by no means a unique property of osteocytes (An et al., 2009). ECM mechanical properties strongly affect the behaviour of cells, which will remodel their internal arrangement and adapt cellular traction forces to match their own mechanical properties to those of the environment. Cells on stiff ECM will pull harder than cells on soft ECM, thereby increasing the tension on force-bearing elements such as F-actin (Trepat et al., 2007). Interestingly, those force-bearing elements are the same as the elements involved in mechanosensing. Hence, ECM properties and the sensitivity of osteocytes to mechanical stimuli are coupled. Physical properties of the ECM are determined by its composition, the organisation of its components and their crosslinking. Cells produce ECM and crosslinkers, and by applying traction forces determine the architecture of ECM molecules and their degradation. Thus, interactions between the cell and the ECM are reciprocal.

Taken together, evidence has been provided demonstrating that the cytoskeleton affects the osteocyte response to stress, implying that the cytoskeleton is directly 
involved in cellular mechanotransduction. Thus, any factor that significantly alters the osteocyte cytoskeleton in principle changes the response of osteocytes to mechanical stimuli, and could thereby affect bone mass.

\section{Molecular signalling and the cytoskeleton in health and disease}

It has been hypothesised often that the osteocytes in the skull have a different sensitivity to mechanical stimulation from the osteocytes in long bones, based on their difference in mechanical environment. Different mechanosensitivity of skull and long bone osteocytes could not be confirmed in vitro (Soejima et al., 2001), where osteocytes were cultured on flat and stiff substrates and unlikely to replicate their site-specific cytoskeletal arrangement and thus mechanosensitivity (Vatsa et al., 2008b). In addition, osteocytes were not grown on their native ECM before being mechanically stimulated. The ECM of skull bones differs significantly from that of long bones and, as already mentioned above, changes in ECM drive cytoskeletal changes and thereby possibly tune osteocyte mechanosensing. Osteoporosis is a bone disease leading to an increased risk of fracture in long bones and vertebrae, but not in the skull bones. Osteoporosis has so far not been connected to a difference in ECM composition or cytoskeletal structure of osteocytes, although it is a long standing hypothesis that changes in osteocyte mechanosensitivity contribute to the imbalance between bone mass and required strength (Bakker et al., 2006).

The osteocyte cytoskeleton might be altered during osteoporosis, since enhanced circulating levels of cytokines are present in postmenopausal osteoporosis, and cytokines can modulate the cytoskeleton in several cell types. Cytokines are also highly expressed during inflammatory diseases such as Crohn's disease and rheumatoid arthritis, and are associated with a loss of bone mass. The cytokines tumour necrosis factor (TNF) $\alpha$ and interleukin (IL)- $1 \beta$ inhibit the increase in NO production and intracellular calcium that is normally observed in cultured osteocytes after application of a mechanical stimulus in the form of a fluid flow (Bakker et al., 2009). TNF $\alpha$ and IL-1 $\beta$ strongly reduce $\mathrm{F}$-actin content, which results in a reduction of osteocyte stiffness as indicated by the elastic moduli determined by twisting magnetic beads attached to the cell, providing a possible mechanism through which inflammation contributes to loss of bone mass (Bakker et al., 2009).

Several bone mass disorders have been linked to mutations in a Wnt receptor and in a Wnt antagonist that is specifically expressed by osteocytes (Little et al., 2002; Van Bezooijen et al., 2007). Hence, molecules involved in Wnt signalling are of interest to the field of osteocyte biology. Wnts are a family of secreted glycoproteins with members that activate various intracellular pathways after binding to frizzled receptors or to a complex comprised of frizzled and LDL receptor-related proteins 5/6 (LRP5/6). The best studied Wnt pathway is the Wnt/ $\beta$-catenin pathway. In the absence of $\mathrm{Wnt}$ ligands, $\beta$-catenin is recruited into a destruction complex and targeted for degradation. A protein named Dishevelled (DVL) is activated upon binding of Wnts to its receptors. Activated DVL recruits a destruction complex containing adenomatous polyposis coli (APC) to the plasma membrane, which prevents degradation of $\beta$-catenin. Because of the incapacitation of the destruction complex after binding of Wnts to the receptors, newly formed $\beta$-catenin accumulates in the cytoplasm and subsequently translocates to the nucleus, where it activates target gene transcription (Lai et al., 2009; Li et al., 2012b). Interestingly, DVL not only plays a central role in coordinating Wnt signalling, it also plays a role in various physiological and morphogenetic processes involving cytoskeletal interactions. DVL colocalises with microtubules and stabilises them (Krylova et al., 2000). APC also interacts with proteins linked to the cytoskeleton, and several observations have highlighted the potential importance of the interaction between APC and microtubules (Matsumoto et al., 2010). $\beta$-catenin has been suggested to alter the sensitivity of bone cells to mechanical loading (Robinson et al., 2006). When MC3T3-E1 osteoblasts are treated with molecules that stabilise $\beta$-catenin and then subjected to mechanical loading, a synergistic up-regulation of Wnt gene expression is observed (Robinson et al., 2006). This suggests that activation of the Wnt signalling cascade enhances the sensitivity of bone cells to mechanical loading. $\beta$-catenin is not only an important component of the Wnt signalling pathway, it also links cadherins (cell-cell adhesion molecules) to the actin cytoskeleton. A model has been proposed in which $\beta$-catenin is launched from its sites of cell-cell attachment towards target genes in the nucleus via cytoskeletal components upon mechanical stimulation (Bidwell and Pavalko, 2010). Although this model is not applicable to osteocytes with regard to $\beta$-catenin since osteocytes do not express cadherins, it would still be applicable to sites of focal adhesions (Bidwell and Pavalko, 2010).

Wnt signalling not only stabilises $\beta$-catenin, it also activates several members of the Rho family of GTPases to affect cellular function (Matsumoto et al., 2010). Rho GTPases and their regulators regulate cytoskeletal remodelling. In light of the role of the cytoskeleton in mechanosensing, it is noteworthy that Wnts may modulate cytoskeletal organisation. Wnt signalling might indeed be an important modulator of the process of mechanoregulated bone adaptation. This is illustrated by the finding that in vivo loading of mouse tibiae results in increased gene expression of Wnts and Wnt target genes including Wnt10B, SFRP1, and Cx 43. In addition, loading of tibiae by means of 4-point bending leads to more bone formation in mice with a dominantly active LRP5 receptor (resulting in a continuous activation of the Wnt signalling cascade) than in wild type mice (Johnson et al., 2004). Similarly, the anabolic response of bone to mechanical loading is enhanced in ulnae of mice lacking the Wnt inhibitor Sfrp3 (Lories et al., 2007). Strikingly, the bones of these mice seemed to be more sensitive to mechanical loading, responding to stimuli that were not sufficient to elicit a response in wild-type mice (Lories et al., 2007). 
Mechanical loading leads to an increase in Wnt protein production by osteocytes, which activates the canonical Wnt signalling pathway in a paracrine fashion (Santos et al., 2009; Tu et al., 2012). Mechanical loading might thus lead to Wnt production by osteocytes, thereby tuning their own sensitivity to mechanical loading in a feedback loop.

The mechanosensitivity of osteocytes has been suggested to diminish with aging, although reports about a diminished anabolic response of bone with age are conflicting (Kohrt, 2001; Srinivassan et al., 2003). There is evidence that aging is associated with changes in the cytoskeleton, which lead to apoptosis and changes in autophagy in chondrocytes, and aging induces changes in the actin and microtubules of primary human osteoblasts (Ankersen et al., 1994). Unfortunately, nothing is known yet about age-related changes in the osteocyte cytoskeleton.

Aging and osteoporosis may not be considered special conditions of illness for bone tissue, considering that no organism is exempt. However, the lack of mechanical load (as in microgravity or bed rest as forms of disuse) may stimulate similar signalling, which in turn provides a special condition towards loss of bone mass and compromised bone structure. Similarly, apoptosis may contribute towards local disuse in terms of the recruitment of osteoclasts towards the vicinity of apoptotic osteocytes by responding to apoptosis-induced signalling (Kogianni et al., 2008). Such a specialised condition could be shortlived as micro-cracks, a pre-condition for osteoclastic resorption, and is also a possible trigger for the onset of bone growth suggested by cellular distribution in the cutting cone (Burger and Klein-Nulend, 1999; Burger et al., 2003).

\section{Conclusion}

The osteocyte cytoskeleton, which ultimately determines cell shape, is a key factor in determining how osteocytes feel stresses, whether by a local or bulk deformation, or via a transfer of forces inside of the cell. As osteocytes orchestrate bone remodelling, any factor affecting the osteocyte cytoskeleton and thereby the osteocyte response to mechanical stimuli, potentially affects bone mass. Accumulated depth in the study of molecular mechanisms involved in bone cell mechanosensing, while slowly completing the "big picture", draws a complex diagram where the role of mechanics is not independent of molecular signalling. Here, the need for computation becomes relevant in linking clusters of possible pathways that promise a practical grasp of correlations between biological function, signalling, and stress sensing. Computation is however a very important tool that will always require experimental verification, that is, predictions ensuing from theory must provide measurable parameters. Given the crucial importance of osteocytes for maintaining a proper resistance against bone fracture, it seems obvious that a much greater knowledge of the molecular mechanisms that govern the adaptive response of osteocytes is needed.

\section{Acknowledgements}

Financial support for the work of R.G. Bacabac was obtained from the Netherlands Organisation For International Cooperation In Higher Education.

\section{References}

Aarden EM, Nijweide PJ, Van der Plas A, Alblas MJ, Mackie EJ, Horton MA, Helfrich MH (1996) Adhesive properties of isolated chick osteocytes in vitro. Bone 18: 305-313.

Addas KM, Schmidt CF, Tang JX (2004) Microrheology of solutions of semiflexible biopolymer filaments using laser tweezers interferometry. Phys Rev E Stat Nonlin Soft Matter Phys 70: 021503.

Ajubi Ajubi NE, Klein-Nulend J, Nijweide PJ, VrijheidLammers T, Alblas MJ, Burger EH (1996) Pulsating fluid flow increases prostaglandin production by cultured chicken osteocytes - a cytoskeleton-dependent process. Biochem Biophys Res Commun 225: 62-68.

Ajubi NE, Klein-Nulend J, Alblas MJ, Burger EH, Nijweide PJ (1999) Signal transduction pathways involved in fluid flow-induced $\mathrm{PGE}_{2}$ production by cultured osteocytes. Am J Physiol 276: E171-E178.

An SS, Kim J, Ahn K, Trepat X, Drake KJ, Kumar S, Ling G, Purington C, Rangasamy T, Kensler TW, Mitzner W, Fredberg JJ, Biswal S (2009) Cell stiffness, contractile stress and the role of extracellular matrix. Biochem Biophys Res Commun 382: 697-703.

Anno T, Sakamoto N, Sato M (2012) Role of nesprin-1 in nuclear deformation in endothelial cells under static and uni-axial stretching conditions. Biochem Biophys Res Commun 424: 94-99.

Ankersen L, Kassem M, Ostergaard LH, Eriksen EF, Clark BF, Rattan SI (1994) Aging of human trabecular osteoblasts in culture. Arch Gerontol Geriatr 19(Suppl 1): 5-12.

Bacabac RG, Smit TH, Mullender MG, Dijcks SJ, Van Loon JJ, Klein-Nulend J (2004) Nitric oxide production by bone cells is fluid shear stress rate dependent. Biochem Biophys Res Commun 315: 823-829.

Bacabac RG, Smit TH, Van Loon JJWA, Zandieh Doulabi B, Helder MN, Klein-Nulend J (2006) Bone cell responses to high-frequency vibration stress: does the nucleus oscillate within the cytoplasm? FASEB J 20: 858864.

Bacabac RG, Mizuno D, Schmidt CF, MacKintosh FC, Van Loon JJWA, Klein-Nulend J, Smit TH (2008) Round versus flat: bone cell morphology, elasticity, and mechanosensing. J Biomech 41: 1590-1598.

Bacabac RG, Van Loon JJWA, Smit TH, Klein-Nulend J (2009) Noise enhances the rapid nitric oxide production by bone cells in response to fluid shear stress. Technol Health Care 17: 57-65.

Bakker AD, Soejima K, Klein-Nulend J, Burger EH (2001) The production of nitric oxide and prostaglandin $\mathrm{E}_{2}$ by primary bone cells is shear stress dependent. $\mathrm{J}$ Biomech 34: 671-677. 
Bakker AD, Klein-Nulend J, Tanck E, Heyligers IC, Albers GH, Lips P, Burger EH (2006) Different responsiveness to mechanical stress of bone cells from osteoporotic versus osteoarthritic donors. Osteoporos Int 17: 827-833.

Bakker AD, Silva VC, Krishnan R, Bacabac RG, Blaauboer ME, Lin YC, Marcantonio RA, Cirelli JA, Klein-Nulend J (2009) Tumor necrosis factor alpha and interleukin-1beta modulate calcium and nitric oxide signalling in mechanically stimulated osteocytes. Arthritis Rheum 60: 3336-3345.

Batra N, Burra S, Siller-Jackson AJ, Gu S, Xia X, Weber GF, DeSimone D, Bonewald LF, Lafer EM, Sprague E, Schwartx MA, Jiang JX (2012) Mechanical stressactivated integrin $\alpha 5 \beta 1$ induces opening of connexin 43 hemichannels. Proc Natl Acad Sci USA 109: 3359-3364.

Berbari NF, O'Connor AK, Haycraft CJ, Yoder BK (2009) The primary cilium as a complex signaling center. Curr Biol 19: R526-R535.

Bidwell JP, Pavalko FM (2010) The load-bearing mechanosome revisited. Clin Rev Bone Miner Metab 8: 213-223.

Bonewald LF (2006) Mechanosensation and transduction in osteocytes. Bonekey Osteovision 3: 7-15.

Bonewald LF (2011) The amazing osteocyte. J Bone Miner Res 26: 229-238.

Bonivtch AR, Bonewald LF, Nicolella DP (2007) Tissue strain amplification at the osteocyte lacuna: a microstructural finite element analysis. J Biomech 40: 2199-2206.

Burger EH, Klein-Nulend J (1999) Mechanotransduction in bone - role of the lacuno-canalicular network. FASEB J 13: S101-S112.

Burger EH, Klein-Nulend J, Smit TH (2002) Strainderived canalicular fluid flow regulates osteoclast activity in a remodeling osteon - a proposal. J Biomech 36: 1453 1459.

Burra S, Nicolella DP, Francis WL, Freitas CJ, Mueschke NJ, Poole K, Jiang JX (2010) Dendritic processes of osteocytes are mechanotransducers that induce the opening of hemichannels. Proc Natl Acad Sci USA 107: 13648-13653.

Burra S, Nicolella DP, Jiang JX (2011) Dark horse in osteocyte biology: Glycocalyx around the dendrites is critical for osteocyte mechanosensing. Commun Integr Biol 4: 48-50.

Davies PF (1995) Flow-mediated endothelial mechanotransduction. Physiol Rev 75: 519-560.

Ducher G, Prouteau S, Courteix D, Benhamou CL (2004) Cortical and trabecular bone at the forearm show different adaptation patterns in response to tennis playing. J Clin Densitom 7: 399-405.

Fritton SP, Weinbaum S (2009) Fluid and solute transport in bone: flow-induced mechanotransduction. Annu Rev Fluid Mech 41: 347-374.

Gardinier JD, Townend CW, Jen KP, Wu Q, Duncan RL, Wang L (2010) In situ permeability measurement of the mammalian lacuno-canalicular system. Bone 46: 1075 1081 .
Gittes F, Schmidt CF (1998) Signals and noise in micromechanical measurements. Methods Cell Biol 55: 129-156.

Globus RK, Bikle DD, Morey-Holton E (1986) The temporal response of bone to unloading. Endocrinology 118: 733-742.

Hoffman BD, GrashoffC, Schwartz MA(2011) Dynamic molecular processes mediate cellular mechanotransduction. Nature 475: 316-323.

Hu J, Chiang LY, Koch M, Lewin GR (2010) Evidence for a protein tether involved in somatic touch. EMBO J 29: 855-867.

Ingber DE (1997) Tensegrity: the architectural basis of cellular mechanotransduction. Ann Rev Physiol 59: 575-599.

Janmey PA (1998) The cytoskeleton and cell signaling: component localization and mechanical coupling. Physiol Rev 78: 763-781.

Johnson ML, Harnish K, Nusse R, Van Hul W (2004) LRP5 and Wnt signaling: a union made for bone. J Bone Miner Res 19: 1749-1757.

Juffer P, Jaspers RT, Lips P, Bakker AD, Klein-Nulend $\mathrm{J}$ (2012) Expression of muscle anabolic and metabolic factors in mechanically loaded MLO-Y4 osteocytes. Am J Physiol Endocrinol Metab 302: E389-E395.

Kamioka H, Sugawara Y, Honjo T, Yamashiro T, Takano-Yamamoto T (2004) Terminal differentiation of osteoblasts to osteocytes is accompanied by dramatic changes in the distribution of actin-binding proteins. $\mathrm{J}$ Bone Miner Res 19: 471-478.

Kardas D, Nackenhorst U, Balzani D (2012) Computational model for the cell-mechanical response of the osteocyte cytoskeleton based on self-stabilizing tensegrity structures. Biomech Model Mechanobiol, in press.

Klein-Nulend J, van der Plas A, Semeins CM, Ajubi NE, Frangos JA, Nijweide PJ, Burger EH (1995a) Sensitivity of osteocytes to biomechanical stress in vitro. FASEB J 9: 441-445.

Klein-Nulend J, Semeins CM, Ajubi NE, Nijweide PJ, Burger EH (1995b) Pulsating fluid flow increases nitric oxide (NO) synthesis by osteocytes but not periosteal fibroblasts - correlation with prostaglandin upregulation. Biochem Biophys Res Commun 217: 640-648.

Knothe-Tate ML, Niederer P, Knothe U (1998) In vivo tracer transport throught the lacunocanalicular system of rat bone in an environment devoid of mechanical loading. Bone 22: 107-117.

Knothe-Tate ML, Steck R, Forwood MR, Niederer P (2000) In vivo demonstration of load-induced fluid flow in the rat tibia and its potential implications for processes associated with functional adaptation. J Exp Biol 203: 2737-2745.

Kogianni G, Mann V, Noble BS (2008) Apoptotic bodies convey activity capable of initiating osteoclastogenesis and localized bone destruction. J Bone Miner Res 23: 915-927.

Kohrt WM (2001) Aging and the osteogenic response to mechanical loading. Int J Sport Nutr Exerc Metab 11(Suppl): S137-S142. 
Krylova O, Messenger MJ, Salinas PC (2000) Dishevelled-1 regulates microtubule stability: a new function mediated by glycogen synthase kinase-3beta. J Cell Biol 151: 83-94.

Kulkarni RN, Bakker AD, Everts V, Klein-Nulend J (2010) Inhibition of osteoclastogenesis by mechanically loaded osteocytes: involvement of MEPE. Calcif Tissue Int 87: 461-468.

Kulkarni RN, Bakker AD, Gruber EV, Chae TD, Veldkamp JBB, Klein-Nulend J, Everts V (2012) MT1MMP modulates the mechanosensitivity of osteocytes. Biochem Biophys Res Commun 417: 824-829.

Lai SL, Chien AJ, Moon RT (2009) Wnt/Fz signaling and the cytoskeleton: potential roles in tumorigenesis. Cell Res 19: 532-545.

Lanyon LE, Hampson WG, Goodship AE, Shah JS (1975) Bone deformation recorded in vivo from strain gauges attached to the human tibial shaft. Acta Orthop Scand 46: 256-268.

Lanyon LE, Rubin CT (1984) Static vs. dynamic loads as an influence on bone remodelling. J Biomech 17: 897905.

Li J, Rose E, Frances D, Sun Y, You L (2012a) Effect of oscillating fluid flow stimulation on osteocyte mRNA expression. J Biomech 45: 247-251.

Li VS, Ng SS, Boersema PJ, Low TY, Karthaus WR, Gerlach JP, Mohammed S, Heck AJ, Maurice MM, Mahmoudi T, Clevers H (2012b) Wnt Signaling through inhibition of $\beta$-catenin degradation in an intact axin1 complex. Cell 149: 1245-1256.

Little RD, Carulli JP, Del Mastro RG, Dupuis J, Osborne M, Folz C, Manning SP, Swain PM, Zhao SC, Eustace B, Lappe MM, Spitzer L, Zweier S, Braunschweiger K, Benchekroun Y, Hu X, Adair R, Chee L, FitzGerald MG, Tulig C, Caruso A, Tzellas N, Bawa A, Franklin B, McGuire S, Nogues X, Gong G, Allen KM, Anisowicz A, Morales AJ, Lomedico PT, Recker SM, Van Eerdewegh P, Recker RR, Johnson ML (2002) A mutation in the LDL receptor-related protein 5 gene results in the autosomal dominant high-bone-mass trait. Am J Hum Genet 70: 1119.

Little R, Muimo R, Robson L, Harris K, Grabowski PS (2011) The transient receptor potential ion channel TRPV6 is expressed at low levels in osteoblasts and has little role in osteoblast calcium uptake. PLoS One 6: e28166.

Litzenberger JB, Kim JB, Tummala P, Jacobs CR (2010) Beta1 integrins mediate mechanosensitive singaling pathways in osteocytes. Calcif Tissue Int 86: 325-332.

Liu C, Zhao Y, Cheung WY, Gandhi R, Wang L, You (2010) Effects of cyclic hydraulic pressure on osteocytes. Bone 46: 1449-1456.

Lombardi ML, Lammerding J (2011) Keeping the LINC: the importance of nucleocytoskeletal coupling in intracellular force transmission and cellular function. Biochem Soc Trans 39: 1729-1734.

Lories RJ, Peeters J, Bakker A, Tylzanowski P, Derese J, Schrooten J, Thomas JT, Luyten FP (2007) Articular cartilage and biomechanical properties of the long bones in Frzb-knockout mice. Arthr Rheum 56: 3881-3883.
Luo G, Cowin SC, Sadegh AM, Arramon YP (1995) Implementation of strain rate as a bone remodeling stimulus. J Biomech Eng 117: 329-338.

Malone AM, Anderson CT, Tummala P, Kwon RY, Johnston TR, Stearns T, Jacobs CR (2007a) Primary cilia mediate mechanosensing in bone cells by a calciumindependent mechanism. Prox Natl Acad Sci USA 104: 13325-13330.

Matsumoto S, Fumoto K, Okamoto T, Kaibuchi K, Kikuchi A(2010) Binding of APC and dishevelled mediates Wnt5a-regulated focal adhesion dynamics in migrating cells. EMBO J 29: 1192-1204.

McGarry JG, Klein-Nulend J, Prendergast PJ (2005a) The effect of cytoskeletal disruption on pulsatile fluid flow-induced nitric oxide and prostaglandin $\mathrm{E}_{2}$ release in osteocytes and osteoblasts. Biochem Biophys Res Commun 330: 341-348.

McGarry JG, Klein-Nulend J, Mullender MG, Prendergast PJ (2005b) A comparison of strain and fluid shear stress in stimulating bone cell responses - a computational and experimental study. FASEB J 19: 482484.

McNamara LM, Majeska RJ, Weinbaum S, Friedrich V, Schaffler MB (2009) Attachment of osteocyte cell processes to the bone matrix. Anat Rec (Hoboken) 292: 355-363.

Mizuno D, Bacabac R, Tardin C, Head D, Schmidt CF (2009) High-resolution probing of cellular force transmission. Phys Rev Lett 102: 16802.

Mosley JR, Lanyon LE (1998) Strain rate as a controlling influence on adaptive modeling in response to dynamic loading of the ulna in growing male rats. Bone 23: 313-318.

Murshid SA, Kamioka H, Ishihara Y, Ando R, Sugawara Y, Takano-Yamamoto T (2007) Actin and microtubule cytoskeletons of the processes of 3D-cultured MC3T3-E1 cells and osteocytes. J Bone Miner Metab 25: 151-158.

Nakashima T, Hayashi M, Fukunaga T, Kurata K, Oh-Hora M, Feng JQ, Bonewald LF, Kodama T, Wutz A, Wagner EF, Penninger JM, Takayanagi H (2011) Evidence for osteocyte regulation of bone homeostasis through RANKL expression. Nat Med 17: 1231-1234.

Noonan KJ, Stevens JW, Tammi R, Tammi M, Hernandez JA, Midura RJ (1996) Spatial distribution of CD44 and hyaluronan in the proximal tibia of the growing rat. J Orthop Res 14: 573-581.

Onal M, Xiong J, Chen X, Thostenson JD, Almeida M, Manolagas SC, O'Brien CA (2012) RANKL expression by B lymphocytes contributes to ovariectomy-induced bone loss. J Biol Chem 287: 29851-29860.

Ozcivici E, Luu YK, Rubin CT, Judex S (2010) Low-level vibrations retain bone marrow's osteogenic potential and augment recovery of trabecular bone during reambulation. PLoS One 5: e11178.

Papaseit C, Pochon N, Tabony J (2000) Microtubule self-organization is gravity-dependent. Proc Natl Acad Sci USA 97: 8364-8368.

Price C, Zhou X, Li W, Wang L (2011) Real-time measurement of solute transport within the lacuno- 
canalicular system of mechanically loaded bone: direct evidence for load-induced fluuid flow. J Bone Miner Res 26: $277-285$.

Reijnders CM, Bravenboer N, Tromp AM, Blankenstein MA, Lips P (2007) Effect of mechanical loading on insulin-like growth factor-I gene expression in rat tibia. J Endocrinol 192: 131-140.

Robinson JA, Chatterjee-Kishore M, Yaworsky PJ, Cullen DM, Zhao W, Li C, Kharode Y, Sauter L, Babij P, Brown EL, Hill AA, Akhter MP, Johnson ML, Recker RR, Komm BS, Bex FJ (2006) Wnt/beta-catenin signaling is a normal physiological response to mechanical loading in bone. J Biol Chem 281: 31720-31728.

Robling AG, Bellido T, Turner CH (2006) Mechanical stimulation in vivo reduces osteocyte expression of sclerostin. J Musculoskelet Neuronal Interact 6: 354.

Rubin CT, Lanyon LE (1987) Kappa Delta Award paper. Osteoregulatory nature of mechanical stimuli: function as a determinant for adaptive remodeling in bone. J Orthop Res 5: 300-310.

Santos A, Bakker AD, Zandieh-Doulabi B, Semeins CM, Klein-Nulend J (2009) Pulsating fluid flow modulates gene expression of proteins involved in Wnt signaling pathways in osteocytes. J Orthop Res 27: 1280-1287.

Santos A, Bakker AD, Zandieh-Doulabi B, de BlieckHogervorst JM, Klein-Nulend J (2010) Early activation of the beta-catenin pathway in osteocytes is mediated by nitric oxide, phosphatidyl inositol-3 kinase/Akt, and focal adhesion kinase. Biochem Biophys Res Commun 391: 364-369.

Srinivasan S, Agans SC, King KA, Moy NY, Poliachik SL, Gross TS (2003) Enabling bone formation in the aged skeleton via rest-inserted mechanical loading. Bone 33: 946-955.

Soejima K, Klein-Nulend J, Semeins CM, Burger EH (2001) Different responsiveness of cells from adult and neonatal mouse bone to mechanical and biochemical challenge. J Cell Physiol 186: 366-370.

Storm C, Pastore JJ, MacKintosh FC, Lubensky TC, Janmey PA (2005) Nonlinear elasticity in biological gels. Nature 435: 191-194.

Sugawara Y, Ando R, Kamioka H, Ishihara Y, Murshid SA, Hashimoto K, Kataoka N, Tsujioka K, Kajiya F, Yamashiro T, Takano-Yamamoto T (2008) The alteration of a mechanical property of bone cells during the process of changing from osteoblasts to osteocytes. Bone 43: 19-24.

Tan SD, de Vries TJ, Kuijpers-Jagtman AM, Semeins CM, Everts V, Klein-Nulend J (2007) Osteocytes subjected to fluid flow inhibit osteoclast formation and bone resorption. Bone 41: 745-751.

Tanaka-Kamioka K, Kamioka H, Ris H, Lim SS (1998) Osteocyte shape is dependent on actin filaments and osteocyte processes are unique actin-rich projections. J Bone Miner Res 13: 1555-1568.

Tatsumi S, Ishii K, Amizuka N, Li M, Kobayashi T, Kohno K, Ito M, Takeshita S, Ikeda K (2007) Targeted ablation of osteocytes induces osteoporosis with defective mechanotransduction. Cell Metab 5: 464-475.

Trepat X, Deng L, An SS, Navajas D, Tschumperlin DJ, Gerthoffer WT, Butler JP, Fredberg JJ (2007) Universal physical responses to stretch in the living cell. Nature 447: 592-595.

Tu X, Rhee Y, Condon KW, Bivi N, Allen MR, Dwyer D, Stolina M, Turner CH, Robling AG, Plotkin LI, Bellido $\mathrm{T}$ (2012) Sost downregulation and local Wnt signaling are required for the osteogenic response to mechanical loading. Bone 50: 209-217.

Turner CH, Forwood MR, Rho JY, Yoshikawa T (1994) Mechanical loading thresholds for lamellar and woven bone formation. J Bone Miner Res 9: 87-97.

Usui T, Maki K, Toki Y, Shibasaki Y, Takanobu H, Takanishi A, Hatcher D, Miller A (2003) Measurement of mechanical strain on mandibular surface with mastication robot: influence of muscle loading direction and magnitude. Orthod Craniofac Res 6(Suppl 1): 163-167; discussion 179-182.

Vandamme K, Holy X, Bensidhoum M, Deschepper M, Logeart-Avramoglou D, Naert I, Duyck J, Petite H (2012) Impaired osteoblastogenesis potential of progenitor cells in skeletal unloading is associated with alterations in angiogenic and energy metabolism profile. Biomed Mater Eng 22: 219-226.

Van Bezooijen RL, Svensson JP, Eefting D, Visser A, van der Horst G, Karperien M, Quax PH, Vrieling H, Papapoulos SE, ten Dijke P, Löwik CW (2007) Wnt but not BMP signaling is involved in the inhibitory action of sclerostin on BMP-stimulated bone formation. J Bone Miner Res 22: 19-28.

Van der Plas A, Nijweide PJ (1992) Isolation and purification of osteocytes. J Bone Miner Res 7: 389-396.

Vatsa A, Semeins CM, Smit TH, Klein-Nulend J (2008a) Paxillin localisation in osteocytes - is it determined by the direction of loading? Biochem Biophys Res Commun 377: 1019-1024.

Vatsa A, Breuls RG, Semeins CM, Salmon PL, Smit TH, Klein-Nulend J (2008b) Osteocyte morphology in fibula and calvaria - is there a role for mechanosensing? Bone 43: 452-458.

Veno P, Nicolella DP, Sivakumar P, Kalajzic I, Rowe D, Harris SE, Bonewald LF, Dallas SL (2006) Live imaging of osteocytes within their lacunae reveals cell body and dendrite motions. J Bone Min Res 21(Suppl 1): S38.

Vezeridis PS, Semeins CM, Chen Q, Klein-Nulend J (2006) Osteocytes subjected to pulsating fluid flow regulate osteoblast proliferation and differentiation. Biochem Biophys Res Commun 348: 1082-1088.

Wang L, Fritton SP, Cowin SC, Weinbaum S (1999) Fluid pressure relaxation depends upon osteonal microstructure: modeling an oscillatory bending experiment. J Biomech 32: 663-672.

Wang Y, McNamara LM, Schaffler MB, Weinbaum S (2007) A model for the role of integrins in flow induced mechanotransduction in osteocytes. Proc Natl Acad Sci USA 104: 15941-15846.

Wu D, Ganatos P, Spray DC, Weinbaum S (2011) On the electrophysiological response of bone cells using a Stokesian fluid stimulus probe for delivery of quantifiable localized picoNewton level forces. J Biomech 44: 17071708.

Wu H, Teng PN, Jayaraman T, Onishi S, Li J, Bannon L, Huang H, Close J, Sfeir C (2011) Dentin matrix protein 
1 (DMP1) signals via cell surface integrin. J Biol Chem 286: 29462-29469.

Xiong J, Onal M, Jilka RL, Weinstein RS, Manolagas SC, O’Brien CA (2011) Matrix-embedded cells control osteoclast formation. Nat Med 17: 1235-1241.

You L, Cowin SC, Schaffler MB, Weinbaum S (2001) A model for strain amplification in the actin cytoskeleton of osteocytes due to fluid drag on pericellular matrix. J Biomech 34: 1375-1386.

You LD, Weinbaum S, Cowin SC, Schaffler MB (2004) Ultrastructure of the osteocyte process and its pericellular matrix. Anat Rec 278A: 505-513.

You L, Temiyasathit S, Lee P, Kim CH, Tummala P, Yao W, Kingery W, Malone AM, Kwon RY, Jacobs CR (2008) Osteocytes as mechanosensors in the inhibition of bone resorption due to mechanical loading. Bone 42: 172-179.
Zang K, Barragan-Adjemian C, Ye L, Kotha S, Dallas M, Lu Y, Zhao S, Harris M, Harris SE, Feng JQ, Bonewald LF (2006) E11/gp38 selective expression in osteocytes: regulation by mechanical strain and role in dendrite elongation. Mol Cell Biol 26: 4539-4552.

Zhang Y, Paul EM, Sathyendra V, Davison A, Sharkey N, Bronson S, Srinivasan S, Gross TS, Donahue HJ (2011) Enhanced osteoclastic resorption and responsiveness to mechanical load in gap junction deficient bone. PLoS One 6: e23516.

Editor's Note: All questions/comments by the reviewers were answered by text changes, hence there is no "Discussion with Reviewers" section. 